\section{PO 7167 IMPROVING USE OF LONG-LASTING INSECTICIDAL NETS IN KAYANGE COMMUNITY OF NORTH-WESTERN BURUNDI: A QUALITY IMPROVEMENT STUDY}

'Desire Habonimana*, ${ }^{1}$ Gabriel Ndayisaba, ${ }^{2}$ Gideon Nimako. ${ }^{1}$ University of Burundi, Bujumbura, Burundi; ${ }^{2}$ University of the Witwatersrand, Johannesburg, South Africa

\subsection{6/bmjgh-2019-EDC.49}

Background The use of long-lasting insecticidal nets (LLINs) for malaria prevention is a cost-effective intervention. WHO recommends universal coverage and use of LLINs. In lowerand middle-income countries, LLINs are provided free of charge but are either not used or misused. Our study sought to improve LLIN use in Kayange community of north-western Burundi by using a model for improvement (MFI).

Methods A one-group, pre/post-test study was conducted. LLIN weekly use was assessed for four weeks before intervention and for another four weeks after intervention. The study was conducted in 96 households. The intervention consisted of testing four different weekly small change actions by using the MFI.

Results Of the 96 households, 83 households (87\%) owned at least one LLIN. However, only 40 households (42\%) owned at least one LLIN for every two people. After intervention, the number of LLINs used increased from 32 to 75 per cent (134\% increase) and the number of persons (general population) sleeping under LLIN from 35 to 73 per cent $(108 \%$ increase). The number of children under 5 years old sleeping under LLIN increased from 31 to 76 per cent $(145 \%$ increase) and the number of pregnant women who slept under LLIN from 43 to 73 per cent (69\% increase). Also, the averages of the number of nights in each week that the general population slept under LLIN increased from 2.13 to 5.11 (140\% increase), children under 5 years old slept under LLIN from 1.68 to 4.78 (184\% increase) and pregnant women slept under LLIN from 1.56 to 4.47 (186\% increase).

Conclusion Our intervention led to significant increase in all outcome indicators. This increase is the result of a combination of an enabling context and an effective implementation of an evidence-based quality improvement intervention. Small tests of change at the community level have the potential for achieving improved outcomes.

\section{PO 8168 CLINICAL UTILITY OF XPERT MTB/RIF ASSAY FOR THE DIAGNOSIS OF EXTRAPULMONARY TUBERCULOSIS IN ETHIOPIA}

\begin{abstract}
${ }^{1}$ Mulualem Tadesse, ${ }^{2}$ Gemeda Abebe, ${ }^{2}$ Danie Yilma, ${ }^{3}$ Ludwig Apers, ${ }^{3}$ Bouke De Jong, ${ }^{3}$ Leen Rigouts. ${ }^{1}$ Mycobacteriology Research Center, Jimma University, Ethiopia; ${ }^{2}$ Jimma University, Ethiopia; ${ }^{3}$ Institute of Tropical Medicine, Antwerp, Belgium
\end{abstract}

\subsection{6/bmjgh-2019-EDC.50}

Background The diagnosis of extrapulmonary tuberculosis (EPTB) is often made on clinical suspicion alone, and many people receive the wrong diagnosis leading to unnecessary TB treatment or poor outcomes from untreated EPTB. In this study, we evaluated the clinical utility of the Xpert MTB/RIF assay on routinely collected extra-pulmonary specimens in Ethiopia.

Methods This study was carried out at Jimma University Specialized Hospital, Southwest Ethiopia from September 2015 to June 2017. Extra-pulmonary specimens were collected from 572 patients clinically suspected of suffering from EPTB. All specimens were tested for $\mathrm{TB}$ by smear-microscopy, culture and Xpert MTB/RIF. The diagnostic accuracy of Xpert MTB/ RIF was calculated compared to a composite reference standard (CRS), composed of liquid culture and anti-TB treatment response.

Results In total, 572 extra-pulmonary specimens (279 lymph node, 159 pleural, 80 peritoneal, 45 cerebrospinal and 9 pericardial fluids) were tested. The pooled sensitivity and specificity of Xpert MTB/RIF were calculated to be $91 \%$ and $90.6 \%$ respectively when compared to culture. The pooled sensitivity of Xpert MTB/RIF was decreased to $75 \%$ and the specificity was improved to $98 \%$ when Xpert MTB/RIF was compared to the CRS. The sensitivities among the specimen types differed markedly. The highest sensitivity was documented for lymph node (90\%), moderate sensitivity for cerebrospinal (53\%), while the sensitivity was lowest for pleural (30\%) and peritoneal (32\%) fluids. Xpert MTB/RIF, in addition, detected rifampicin resistance in 13 patients in perfect agreement with line probe assay.

Conclusion Our study showed that Xpert MTB/RIF is likely to be of greatest utility when testing lymph node specimens. A negative Xpert MTB/RIF result on fluid specimens does not exclude the diagnosis of EPTB and patients with a high clinical probability of EPTB should be started on anti-TB treatment.

\section{PO 8171 PREDICTORS OF LOSS TO FOLLOW-UP IN ART- COMMENCED PATIENTS IN NIGERIA: A 13-YEAR REVIEW (2004-2017)}

Ahmad Aliyu*, Babatunde Adelekan, Nifarta Andrew, Eunice Ekong, Stephen Dapiap, Fati Murtala-Ibrahim, Nicaise Ndembi, Iboro Nta, Charles Mensah, Patrick Dakum. Institute of Human Virology, Nigeria

\subsection{6/bmjgh-2019-EDC.51}

Background Expanded access to antiretroviral therapy (ART) has improved HIV outcomes in Nigeria. However, increasing rates of patients lost to follow-up (LTFU) is threatening the achievement of the UNAIDS treatment targets to treat $90 \%$ of HIV-diagnosed patients and attain $90 \%$ viral suppression amongst those on treatment. Therefore, this retrospective cross-sectional study is aimed at identifying correlates and predictors of loss to follow-up in ART-commenced patients in a large HIV programme in Nigeria.

Methods Records of all patients who started ART from 2004 to 2017 of 432 PEPFAR-supported facilities across 10 states in Nigeria were used for this study. Univariate, bivariate and multivariate analysis using frequencies, percentages, chi-square and logistic regression was conducted using STATA version 14 to determine occurrence, correlates and predictors of LTFU. Results Among all 245257 ever-enrolled-on-ART patients within the review period, 150191 patients (61.2\%) remained on treatment while 75041 (30.6\%) were LTFU. Patients were significantly more likely to be LTFU when non-pregnant female (OR: 4.55,p<0.001); on $\geq 3$ monthly ARV refills (OR: 1.32, $\mathrm{p}<0.001)$; with unsuppressed viral loads on ART (OR: 4.52, $\mathrm{p}<0.001)$; adult on second-line regimen $(\mathrm{OR}$ : $1.23 \mathrm{p}<0.001$ ); paediatric on first-line regimen (OR: 1.70, $\mathrm{p}<0.001$ ); 10-14 years (OR: 2.99, $\mathrm{p}<0.001$ ); and $\geq 65$ years (OR: 1622.84, p<0.001).

Conclusion Despite increasing access to ART, LTFU is still a challenge in the HIV programme in Nigeria with gender, type of regimen, age, unsuppressed viral load, duration of ARV 